ILIRIAS JOURNAL OF MATHEMATICS

ISSN: 2334-6574, URL: WWW.ILIRIAS.COM/IJM

Volume 9 Issue 1(2021), Pages 23-34.

DOI:10.54379/IJM-2021-1-2

\title{
ON INVARIANT ARITHMETIC STATISTICALLY CONVERGENCE VIA WEIGHTED DENSITY
}

\author{
MUALLA BIRGÜL HUBAN, MEHMET GÜRDAL
}

\begin{abstract}
In this paper, our concern is to introduce the concepts of invariant arithmetic convergence, invariant arithmetic statistically convergence and lacunary invariant arithmetic statistically convergence using weighted density via Orlicz function $\widetilde{\phi}$. Finally, we give some relations between lacunary invariant arithmetic statistical $\widetilde{\phi}$-convergence and invariant arithmetic statistical $\widetilde{\phi}$-convergence via weighted density.
\end{abstract}

\section{INTRODUCTION}

The idea of statistical convergence was first introduced by Fast [12. It became a notable topic in summability theory after the work of Fridy [14] and Šalát 34. Statistical convergence has several applications in different fields of mathematics like number theory, trigonometric series, summability theory, statistics and probability theory, measure theory, optimization, approximation theory and rough set theory. Since then several generalizations and applications of this concept have been investigated by various authors, namely Fridy and Orhan [15, Gürdal and Huban [17, Nabiev et al. [29], Schaefer [40, and many others (see 66, 8, 10, 11, 16, 17, 18, 19, 22, 36, 38,). Also, the readers should refer to the monographs [3, 4] and [28] for the background on the sequence spaces and related topics. The idea of arithmetic convergence was introduced by Ruckle 33. The studies on arithmetic convergence and related results can be found in [23, 24, 25, 41, 42].

In this study, the concepts of invariant arithmetic weight $g$-statistically $\widetilde{\phi}$-convergence, lacunary invariant arithmetic $\widetilde{\phi}$-convergence of weight $g$, invariant arithmetic weight $g$-statistically $\widetilde{\phi}$-convergence and lacunary invariant arithmetic weight $g$-statistically $\widetilde{\phi}$-convergence have been investigated. We also establish some relations between lacunary invariant arithmetic weight $g$-statistical $\widetilde{\phi}$-convergence and invariant arithmetic weight $g$-statistical $\widetilde{\phi}$-convergence.

2000 Mathematics Subject Classification. 40A05, 40A99, 46A70, 46A99.

Key words and phrases. Weighted density; Lacunary sequence; Statistical convergence; invariant arithmetic convergence; $\widetilde{\phi}$-convergence.

(C)2021 Ilirias Research Institute, Prishtinë, Kosovë.

Submitted June 25, 2021. Published September 2, 2021.

Communicated by S.A. Mohiuddine. 


\section{Definitions and Notations}

Statistical convergence depends on the natural density of subsets of the set $\mathbb{N}$ of positive integers. The natural density $\delta(A)$ of a subset $A$ of $\mathbb{N}$ is defined by

$$
\delta(A)=\lim _{t \rightarrow \infty} t^{-1}|\{m \leq t: m \in A\}|
$$

where the vertical bars indicate the number of elements in the enclosed set. A sequence $\left(x_{m}\right)$ is said to be statistically convergent to $\ell$ if for each $\varepsilon>0$,

$$
\lim _{t \rightarrow \infty} t^{-1}\left|\left\{m \leq t:\left|x_{m}-\ell\right| \geq \varepsilon\right\}\right|=0 .
$$

In several literary works, statistical convergence of any real sequence is identified relatively to absolute value. While we have known that the absolute value of real numbers is special of an Orlicz function [31, that is, a function $\widetilde{\phi}: \mathbb{R} \rightarrow \mathbb{R}$ in such a way that it is even, non-decreasing on $\mathbb{R}^{+}$, continuous on $\mathbb{R}$, and satisfying

$$
\widetilde{\phi}(x)=0 \text { if and only if } x=0 \text { and } \widetilde{\phi}(x) \rightarrow \infty \text { as } x \rightarrow \infty .
$$

Further, an Orlicz function $\widetilde{\phi}: \mathbb{R} \rightarrow \mathbb{R}$ is said to satisfy the $\triangle_{2}$ condition, if there exists an positive real number $M$ such that $\widetilde{\phi}(2 x) \leq M . \widetilde{\phi}(x)$ for every $x \in \mathbb{R}^{+}$(also see [19, 37]). In [31, Rao and Ren describes some important applications of Orlicz functions in many areas such as economics, stochastic problems etc. The reader can also refer to the paper [9] and recent monograph [4 related with various ways to generalize Orlicz sequence spaces systematically and investigate several structural properties of such spaces.

By a lacunary sequence we mean an increasing integer sequence $\theta=\left\{k_{r}\right\}$ such that $k_{0}=0$ and $h_{r}=k_{r}-k_{r-1} \rightarrow \infty$ as $r \rightarrow \infty$. Throughout this paper the intervals determined by $\theta$ will be denoted by $I_{r}=\left(k_{r-1}, k_{r}\right]$, and ratio $\frac{k_{r}}{k_{r-1}}$ will be abbreviated by $q_{r}$. The space of lacunary convergent sequence $N_{\theta}$ was introduced by Freedman [13. In [15] the relation between lacunary statistical convergence and statistical convergence was established among other things. And the notion of lacunary convergence has been investigated by many authors [7, 21, 26].

Several authors have studied invariant convergent sequences (see, [20, 30, 32]).

Let $\sigma$ be a mapping of the positive integers into themselves. A continuous linear functional $\varphi$ on $\ell_{\infty}$, the space of real bounded sequences, is said to be an invariant mean or a $\sigma$-mean if it satisfies following conditions:

(i) $\varphi(x) \geq 0$, when the sequence $x=\left(x_{n}\right)$ has $x_{n} \geq 0$ for all $n$,

(ii) $\varphi(e)=1$, where $e=(1,1,1, \ldots)$ and

(iii) $\varphi\left(x_{\sigma(n)}\right)=\varphi\left(x_{n}\right)$ for all $x \in \ell_{\infty}$.

The mapping $\sigma$ are assumed to be one-to-one and such that $\sigma^{m}(n) \neq n$ for all positive integers $n$ and $m$, where $\sigma^{m}(n)$ denotes the $m$ th iterate of the mapping $\sigma$ at $n$. Thus, $\varphi$ extends the limit functional on $c$, the space of convergent sequences, in the sense that $\varphi\left(x_{n}\right)=\lim x_{n}$ for all $x \in c$. In the case $\sigma$ is translation mappings $\sigma(n)=n+1$, the $\sigma$-mean is often called a Banach limit. The space $V_{\sigma}$, the set of bounded sequences whose invariant means are equal, is the set of almost convergent sequences.

A set $E$ of positive integers said to have uniform invariant density of zero if and only if the number of elements of $E$ which lie in the set $\left\{\sigma(m), \sigma^{2}(m), \ldots, \sigma^{n}(m)\right\}$ is $o(n)$ as $n \rightarrow \infty$, uniformly in $m$.

The idea of arithmetic convergence was firstly originated by Ruckle 33. A sequence $x=\left(x_{m}\right)$ is called arithmetically convergent if for each $\varepsilon>0$ there is 
an integer $n$ such that for every integer $m$ we have $\left|x_{m}-x_{\langle m, n\rangle}\right|<\varepsilon$, where the symbol $\langle m, n\rangle$ denotes the greatest common divisor of two integers $m$ and $n$. We denote the sequence space of all arithmetic convergent sequence by $A C$.

The arithmetic statistically convergence and lacunary arithmetic statistically convergence was examined by Yaying and Hazarika [42].

Definition 2.1. A sequence $x=\left(x_{m}\right)$ is said to be arithmetic statistically convergent if for $\varepsilon>0$, there is an integer $n$ such that

$$
\lim _{t \rightarrow \infty} \frac{1}{t}\left|\left\{m \leq t:\left|x_{m}-x_{\langle m, n\rangle}\right| \geq \varepsilon\right\}\right|=0 .
$$

We shall use ASC to denote the set of all arithmetic statistical convergent sequences.

Thus, for $\varepsilon>0$ and integer $n$

$$
A S C=\left\{x=\left(x_{m}\right): \lim _{t \rightarrow \infty} \frac{1}{t}\left|\left\{m \leq t:\left|x_{m}-x_{\langle m, n\rangle}\right| \geq \varepsilon\right\}\right|=0\right\} .
$$

We shall write $A S C-\lim x_{m}=x_{\langle m, n\rangle}$ to denote the sequence $\left(x_{m}\right)$ is arithmetic statistically convergent to $x_{\langle m, n\rangle}$.

Definition 2.2. A sequence $x=\left(x_{m}\right)$ is said to be lacunary arithmetic statistically convergent if for $\varepsilon>0$ there is an integer $n$ such that

$$
\lim _{r \rightarrow \infty} \frac{1}{h_{r}} \mid\left\{m \in I_{r}:\left|x_{m}-x_{\langle m, n\rangle}\right| \geq \varepsilon\right\}=0 .
$$

We shall write

$$
A S C_{\theta}=\left\{x=\left(x_{m}\right): \lim _{r \rightarrow \infty} \frac{1}{h_{r}} \mid\left\{m \in I_{r}:\left|x_{m}-x_{\langle m, n\rangle}\right| \geq \varepsilon\right\}=0\right\} .
$$

We will use $A S C_{\theta}-\lim x_{m}=x_{\langle m, n\rangle}$ to denote the sequence $\left(x_{m}\right)$ is lacunary arithmetic statistically convergent to $x_{\langle m, n\rangle}$.

In the recent times in [2], using the density of weight $g$ where $g: \mathbb{N} \rightarrow[0, \infty)$ is a function with property that $\lim _{t \rightarrow \infty} g(t)=\infty$ and $\frac{t}{g(t)} \nrightarrow 0$ as $t \rightarrow \infty$, the concept of natural density was extended as follows: The upper density of weight $g$ was defined by

$$
\bar{\delta}_{g}(A)=\lim _{t \rightarrow \infty} \sup \frac{A(1, t)}{g(t)}
$$

for $A \subset \mathbb{N}$, where $A(1, t)$ denotes the number of elements in $A \cap[1, t]$. The lower density of weight $g$ is defined in a similar manner. Then, the family

$$
\mathcal{I}_{g}=\left\{A \subset \mathbb{N}: \bar{\delta}_{g}(A)=0\right\}
$$

creates an ideal. It was seen in [2] that $\mathbb{N} \in \mathcal{I}_{g}$ iff $\frac{t}{g(t)} \rightarrow 0$ as $t \rightarrow \infty$. Furthermore, we suppose that $t / g(t) \nrightarrow 0$ as $t \rightarrow \infty$ so that $\mathbb{N} \notin \mathcal{I}_{g}$ and $\mathcal{I}_{g}$ is a proper admissible ideal of $\mathbb{N}$. We denote by $G$ the collection of such weight functions $g$ satisfying the above properties (see [1, 5, 27, 35, 39]).

If we take the function $g(t)=t$ we obtain the usual statistical convergence. It is clear that every convergent sequence is also weight $g$-statistical convergent. But the converse is not true in general. 
Example 2.3. Let us define the function $g(t)=3 t$ and the sequence as

$$
x_{m}=\left\{\begin{array}{lc}
1, & m=t^{2}, t \in \mathbb{N} \\
0, & m \neq t^{2}
\end{array}\right.
$$

Then $\frac{1}{3 t}\left|\left\{m \leq t: x_{m} \neq 0\right\}\right| \leq \frac{\sqrt{t}}{3 t}$. Hence, $g-s t-\lim x_{m}=0$.

As a natural consequence we can introduce the following definition.

Definition 2.4. Let $x=\left(x_{m}\right)$ be a real valued sequence. $x$ is arithmetic weighted $g$-statistical $\widetilde{\phi}$-convergent if for any given $\varepsilon>0$ and integer $n$

$$
\lim _{t \rightarrow \infty} \frac{\left|\left\{m \leq t: \widetilde{\phi}\left(x_{m}-x_{\langle m, n\rangle}\right) \geq \varepsilon\right\}\right|}{g(t)}=0 .
$$

In this case we write st $(\widetilde{\phi})^{g}-\lim x_{m}=x_{\langle m, n\rangle}$.

Remark. If we take the functions $\widetilde{\phi}(x)=|x|$ and $g(t)=t$, then convergent concepts in above definition coincide with arithmetic statistically convergent in [42].

\section{Main Results}

Following the above definitions and results, we aim in this section to introduce some new notions of invariant arithmetic weight $g$-statistical $\widetilde{\phi}$-convergent. In addition to these definition, natural inclusion theorems shall also be presented.

Definition 3.1. A sequence $x=\left(x_{m}\right)$ is said to be invariant arithmetic weight $g$-convergent if for an integer $n$

$$
\lim _{t \rightarrow \infty} \frac{1}{g(t)} \sum_{m=1}^{t} x_{\sigma^{m}(u)}=x_{\langle m, n\rangle},
$$

uniformly in $u=1,2, \ldots$

In this case we write $x_{m} \rightarrow x_{\langle m, n\rangle}\left(A V_{\sigma}\right)^{g}$ and the set of all invariant arithmetic weight $g$-convergent sequences will be demostrated by $\left(A V_{\sigma}\right)^{g}$.

Definition 3.2. Let $\widetilde{\phi}: \mathbb{R} \rightarrow \mathbb{R}$ be an Orlicz function. A sequence $x=\left(x_{m}\right)$ is said to be strongly invariant arithmetic $\widetilde{\phi}$-convergent of weight $g$ if for an integer $n$

$$
\lim _{t \rightarrow \infty} \frac{1}{g(t)} \sum_{m=1}^{t} \widetilde{\phi}\left(x_{\sigma^{m}(u)}-x_{\langle m, n\rangle}\right)=0,
$$

uniformly in $u=1,2, \ldots$

In this case we write $x_{m} \rightarrow x_{\langle m, n\rangle}\left[A V_{\sigma}\right](\widetilde{\phi})^{g}$ to denote the sequence $\left(x_{m}\right)$ is strongly invariant arithmetic $\widetilde{\phi}$-convergent of weight $g$ to $x_{\langle m, n\rangle}$ and the set of all invariant arithmetic $\widetilde{\phi}$-convergent of weight $g$ sequences will be demostrated by $\left[A V_{\sigma}\right](\widetilde{\phi})^{g}$.

Definition 3.3. Let $\widetilde{\phi}: \mathbb{R} \rightarrow \mathbb{R}$ be an Orlicz function. A sequence $x=\left(x_{m}\right)$ is said to be invariant arithmetic weight $g$-statistically $\widetilde{\phi}$-convergent if for every $\varepsilon>0$, there is an integer $n$ such that

$$
\lim _{t \rightarrow \infty} \frac{1}{g(t)}\left|\left\{m \leq t: \widetilde{\phi}\left(x_{\sigma^{m}(u)}-x_{\langle m, n\rangle}\right) \geq \varepsilon\right\}\right|=0,
$$


uniformly in $u=1,2, \ldots$.

We shall use $A S_{\sigma} C(\widetilde{\phi})^{g}$ to denote the set of all invariant arithmetic weight $g$-statistical $\widetilde{\phi}$-convergent sequences. Thus, we define

$$
A S_{\sigma} C(\widetilde{\phi})^{g}=\left\{x=\left(x_{m}\right): \text { for some } x_{\langle m, n\rangle}, A S_{\sigma} C(\widetilde{\phi})^{g}-\lim x_{m}=x_{\langle m, n\rangle}\right\} .
$$

In this case we write $A S_{\sigma} C(\widetilde{\phi})^{g}-\lim x_{m}=x_{\langle m, n\rangle}\left(A S_{\sigma} C\right)$.

For the next result we assume the convex Orlicz function which satisfies $\Delta_{2}$ condition.

Theorem 3.4. Let $\widetilde{\phi}: \mathbb{R} \rightarrow \mathbb{R}$ be an Orlicz function and $x=\left(x_{m}\right)$ and $y=\left(y_{m}\right)$ be two sequences.

(i) If $A S_{\sigma} C(\widetilde{\phi})^{g}-\lim x_{m}=x_{\langle m, n\rangle}$ and $\alpha$ is any real constant, then $A S_{\sigma} C(\widetilde{\phi})^{g}-$ $\lim \left(\alpha x_{m}\right)=\alpha x_{\langle m, n\rangle}$.

(ii) If $A S_{\sigma} C(\widetilde{\phi})^{g}-\lim x_{m}=x_{\langle m, n\rangle}$ and $A S_{\sigma} C(\widetilde{\phi})^{g}-\lim y_{m}=y_{\langle m, n\rangle}$, then $A S_{\sigma} C(\widetilde{\phi})^{g}-\lim \left(x_{m}+y_{m}\right)=\left(x_{\langle m, n\rangle}+y_{\langle m, n\rangle}\right)$.

Proof. Since $\widetilde{\phi}$ satisfies the $\Delta_{2}$-condition, then there exists $M>0$ such that $\widetilde{\phi}(2 x) \leq M . \widetilde{\phi}(x)$, for every $x \in \mathbb{R}$.

(i) The result is obvious when $\alpha=0$. Let $q \in \mathbb{N}$ such that $|\alpha| \leq 2^{q}$ and $A S_{\sigma} C(\widetilde{\phi})^{g}-\lim x_{m}=x_{\langle m, n\rangle}$, then for integer $n$

$$
\lim _{t \rightarrow \infty} \frac{1}{g(t)}\left|\left\{m \leq t: \widetilde{\phi}\left(x_{\sigma^{m}(u)}-x_{\langle m, n\rangle}\right)<\varepsilon\right\}\right|=1 .
$$

Let us consider such $m$ for which $\widetilde{\phi}\left(x_{\sigma^{m}(u)}-x_{\langle m, n\rangle}\right)<\frac{\varepsilon}{2^{q}}$, then

$$
\begin{aligned}
\widetilde{\phi}\left(\alpha x_{\sigma^{m}(u)}-\alpha x_{\langle m, n\rangle}\right) & =\widetilde{\phi}\left(|\alpha|\left(x_{\sigma^{m}(u)}-x_{\langle m, n\rangle}\right)\right) \leq \widetilde{\phi}\left(2^{q}\left(x_{\sigma^{m}(u)}-x_{\langle m, n\rangle}\right)\right) \\
& \leq 2^{q} \widetilde{\phi}\left(x_{\sigma^{m}(u)}-x_{\langle m, n\rangle}\right) \leq \varepsilon .
\end{aligned}
$$

Thus for integer $n$

$$
\lim _{t \rightarrow \infty} \frac{1}{g(t)}\left|\left\{m \leq t: \widetilde{\phi}\left(\alpha x_{\sigma^{m}(u)}-\alpha x_{\langle m, n\rangle}\right)<\varepsilon\right\}\right|=1,
$$

i.e.

$$
\lim _{t \rightarrow \infty} \frac{1}{g(t)}\left|\left\{m \leq t: \widetilde{\phi}\left(\alpha x_{\sigma^{m}(u)}-\alpha x_{\langle m, n\rangle}\right) \geq \varepsilon\right\}\right|=0 .
$$

Hence $A S_{\sigma} C(\widetilde{\phi})^{g}-\lim \left(\alpha x_{m}\right)=\alpha x_{\langle m, n\rangle}$.

(ii) Let $A S_{\sigma} C(\widetilde{\phi})^{g}-\lim x_{m}=x_{\langle m, n\rangle}$ and $A S_{\sigma} C(\widetilde{\phi})^{g}-\lim y_{m}=y_{\langle m, n\rangle}$, i.e.

$$
\begin{aligned}
& \lim _{t \rightarrow \infty} \frac{1}{g(t)}\left|\left\{m \leq t: \widetilde{\phi}\left(x_{\sigma^{m}(u)}-x_{\langle m, n\rangle}\right) \geq \varepsilon\right\}\right| \\
& =0=\lim _{t \rightarrow \infty} \frac{1}{g(t)}\left|\left\{m \leq t: \widetilde{\phi}\left(y_{\sigma^{m}(u)}-y_{\langle m, n\rangle}\right) \geq \varepsilon\right\}\right|,
\end{aligned}
$$


or

$$
\begin{aligned}
& \lim _{t \rightarrow \infty} \frac{1}{g(t)}\left|\left\{m \leq t: \widetilde{\phi}\left(x_{\sigma^{m}(u)}-x_{\langle m, n\rangle}\right)<\varepsilon\right\}\right| \\
& =1=\lim _{t \rightarrow \infty} \frac{1}{g(t)}\left|\left\{m \leq t: \widetilde{\phi}\left(y_{\sigma^{m}(u)}-y_{\langle m, n\rangle}\right)<\varepsilon\right\}\right|,
\end{aligned}
$$

for integer $n$. Let us consider such $m$ for which both of $\widetilde{\phi}\left(x_{\sigma^{m}(u)}-x_{\langle m, n\rangle}\right)<\frac{\varepsilon}{2 M}$ and $\widetilde{\phi}\left(y_{\sigma^{m}(u)}-y_{\langle m, n\rangle}\right)<\frac{\varepsilon}{2 M}$ are true. Hence we have

$$
\begin{aligned}
& \widetilde{\phi}\left(\left(x_{\sigma^{m}(u)}+y_{\sigma^{m}(u)}\right)-\left(x_{\langle m, n\rangle}+y_{\langle m, n\rangle}\right)\right) \\
& =\widetilde{\phi}\left(\left(x_{\sigma^{m}(u)}-x_{\langle m, n\rangle}\right)+\left(y_{\sigma^{m}(u)}-y_{\langle m, n\rangle}\right)\right) \\
& \leq \widetilde{\phi}\left(2\left(x_{\sigma^{m}(u)}-x_{\langle m, n\rangle}\right)+2\left(y_{\sigma^{m}(u)}-y_{\langle m, n\rangle}\right)\right) \\
& \leq M \cdot\left(\widetilde{\phi}\left(x_{\sigma^{m}(u)}-x_{\langle m, n\rangle}\right)+\widetilde{\phi}\left(y_{\sigma^{m}(u)}-y_{\langle m, n\rangle}\right)\right) \leq \varepsilon .
\end{aligned}
$$

Thus for integer $n$

$$
\lim _{t \rightarrow \infty} \frac{1}{g(t)}\left|\left\{m \leq t: \widetilde{\phi}\left(\left(x_{\sigma^{m}(u)}+y_{\sigma^{m}(u)}\right)-\left(x_{\langle m, n\rangle}+y_{\langle m, n\rangle}\right)\right)<\varepsilon\right\}\right|=1
$$

i.e.

$$
\lim _{t \rightarrow \infty} \frac{1}{g(t)}\left|\left\{m \leq t: \widetilde{\phi}\left(\left(x_{\sigma^{m}(u)}+y_{\sigma^{m}(u)}\right)-\left(x_{\langle m, n\rangle}+y_{\langle m, n\rangle}\right)\right) \geq \varepsilon\right\}\right|=0 .
$$

Hence $A S_{\sigma} C(\widetilde{\phi})^{g}-\lim \left(x_{m}+y_{m}\right)=\left(x_{\langle m, n\rangle}+y_{\langle m, n\rangle}\right)$.

Definition 3.5. Let $\widetilde{\phi}: \mathbb{R} \rightarrow \mathbb{R}$ be an Orlicz function. A sequence $x=\left(x_{m}\right)$ is called to be lacunary invariant arithmetic weight $g$-statistical $\widetilde{\phi}$-convergent if for every $\varepsilon>0$, there is an integer $n$ such that

$$
\lim _{r \rightarrow \infty} \frac{1}{g\left(h_{r}\right)}\left|\left\{m \in I_{r}: \widetilde{\phi}\left(x_{\sigma^{m}(u)}-x_{\langle m, n\rangle}\right) \geq \varepsilon\right\}\right|=0,
$$

uniformly in $u=1,2, \ldots$.

We shall use $A S_{\sigma \theta} C(\widetilde{\phi})^{g}$ to indicate the set of all lacunary invariant arithmetic weight $g$-statistical $\widetilde{\phi}$-convergent sequences. Thus, we define

$$
A S_{\sigma \theta} C(\widetilde{\phi})^{g}=\left\{x=\left(x_{m}\right) \text { : for some } x_{\langle m, n\rangle}, A S_{\sigma \theta} C(\widetilde{\phi})^{g}-\lim x_{m}=x_{\langle m, n\rangle}\right\} \text {. }
$$

In this case we write $A S_{\sigma \theta} C(\widetilde{\phi})^{g}-\lim x_{m}=x_{\langle m, n\rangle}$ or $x_{m} \rightarrow x_{\langle m, n\rangle}\left(A S_{\sigma \theta} C\right)_{(\widetilde{\phi})^{g}}$.

By using the same techniques as in Theorem 3.4, we can prove the following theorem.

Theorem 3.6. Let $\widetilde{\phi}: \mathbb{R} \rightarrow \mathbb{R}$ be an Orlicz function and $x=\left(x_{m}\right)$ and $y=\left(y_{m}\right)$ be two sequences.

(i) If $A S_{\sigma \theta} C(\widetilde{\phi})^{g}-\lim x_{m}=x_{\langle m, n\rangle}$ and $\alpha$ is any real constant, then $A S_{\sigma \theta} C(\widetilde{\phi})^{g}-$ $\lim \left(\alpha x_{m}\right)=\alpha x_{\langle m, n\rangle}$.

(ii) If $A S_{\sigma \theta} C(\widetilde{\phi})^{g}-\lim x_{m}=x_{\langle m, n\rangle}$ and $A S_{\sigma \theta} C(\widetilde{\phi})^{g}-\lim y_{m}=y_{\langle m, n\rangle}$, then $A S_{\sigma \theta} C(\widetilde{\phi})^{g}-\lim \left(x_{m}+y_{m}\right)=\left(x_{\langle m, n\rangle}+y_{\langle m, n\rangle}\right)$. 
Definition 3.7. Let $\widetilde{\phi}: \mathbb{R} \rightarrow \mathbb{R}$ be an Orlicz function. A sequence $x=\left(x_{m}\right)$ is said to be strongly lacunary invariant arithmetic $\widetilde{\phi}$-convergent of weight $g$ if for an integer $n$

$$
\lim _{r \rightarrow \infty} \frac{1}{g\left(h_{r}\right)} \sum_{m \in I_{r}} \widetilde{\phi}\left(x_{\sigma^{m}(u)}-x_{\langle m, n\rangle}\right)=0,
$$

uniformly in $u=1,2, \ldots$

In this case we write $x_{m} \rightarrow x_{\langle m, n\rangle}\left(A L_{\sigma \theta}\right)(\widetilde{\phi})^{g}$ to denote the sequence $\left(x_{m}\right)$ is strongly lacunary invariant arithmetic $\widetilde{\phi}$-convergent of weight $g$ to $x_{\langle m, n\rangle}$ and the set of all strongly lacunary invariant arithmetic $\widetilde{\phi}$-convergent of weight $g$ sequences will be denoted $\left(A L_{\sigma \theta}\right)(\widetilde{\phi})^{g}$.

Definition 3.8. Let $\widetilde{\phi}: \mathbb{R} \rightarrow \mathbb{R}$ be an Orlicz function. A sequence $\left\{x_{m}\right\}$ is said to be $\widetilde{\phi}$-bounded if there exists $M>0$ such that $\widetilde{\phi}\left(x_{m}\right) \leq M$ for $m \in \mathbb{N}$. We denote the space of all bounded sequences by $\ell_{\infty}^{\widetilde{\phi}}$.

Now, we give some inclusion relations between $A L_{\sigma \theta}(\widetilde{\phi})^{g}$-convergence and $A S_{\sigma \theta} C(\widetilde{\phi})^{g}$ convergence and demonstrate that these are equivalent for bounded sequences. We also study relation between $A S_{\sigma \theta} C(\widetilde{\phi})^{g}$-convergence and $A S_{\sigma} C(\widetilde{\phi})^{g}$-convergence.

Theorem 3.9. Let $\widetilde{\phi}: \mathbb{R} \rightarrow \mathbb{R}$ be an Orlicz function and $\theta=\left\{k_{r}\right\}$ be a lacunary sequence. Then, the following statements hold:

(i) $x_{m} \rightarrow x_{\langle m, n\rangle}\left(A L_{\sigma \theta}\right)(\widetilde{\phi})^{g}$ implies $x_{m} \rightarrow x_{\langle m, n\rangle}\left(A S_{\sigma \theta} C\right)(\widetilde{\phi})^{g}$,

(ii) $x_{m} \in \ell_{\infty}^{\widetilde{\phi}}$ and $x_{m} \rightarrow x_{\langle m, n\rangle}\left(A S_{\sigma \theta} C\right)(\widetilde{\phi})^{g}$ imply $x_{m} \rightarrow x_{\langle m, n\rangle}\left(A L_{\sigma \theta}\right)(\widetilde{\phi})^{g}$,

(iii) $\left(A S_{\sigma \theta} C\right)(\widetilde{\phi})^{g} \cap \ell_{\infty}=\left(A L_{\sigma \theta}\right)(\widetilde{\phi})^{g}$.

Proof. (i) Let $\varepsilon>0$ and $x_{m} \rightarrow x_{\langle m, n\rangle}\left(A L_{\sigma \theta}\right)(\widetilde{\phi})^{g}$. Then, we can write

$$
\begin{aligned}
& \sum_{m \in I_{r}} \widetilde{\phi}\left(x_{\sigma^{m}(u)}-x_{\langle m, n\rangle}\right) \geq \sum_{\substack{m \in I_{r} \\
\tilde{\phi}\left(x_{\sigma^{m}}(u)-x_{\langle m, n\rangle}\right) \geq \varepsilon}} \widetilde{\phi}\left(x_{\sigma^{m}(u)}-x_{\langle m, n\rangle}\right) \\
& \geq \varepsilon \cdot\left|\left\{m \in I_{r}: \widetilde{\phi}\left(x_{\sigma^{m}(u)}-x_{\langle m, n\rangle}\right) \geq \varepsilon\right\}\right|
\end{aligned}
$$

and so

$$
\frac{1}{\varepsilon g\left(h_{r}\right)} \sum_{t \in I_{r}} \widetilde{\phi}\left(x_{\sigma^{m}(u)}-x_{\langle m, n\rangle}\right) \geq \frac{1}{g\left(h_{r}\right)}\left|\left\{t \in I_{r}: \widetilde{\phi}\left(x_{\sigma^{m}(u)}-x_{\langle m, n\rangle}\right) \geq \varepsilon\right\}\right| .
$$

which gives the result.

(ii) Suppose that $x_{m} \rightarrow x_{\langle m, n\rangle}\left(A S_{\sigma \theta} C\right)(\widetilde{\phi})^{g}$ and $x_{m} \in \ell_{\infty}^{\widetilde{\phi}}$. If $x_{m} \in \ell_{\infty}^{\widetilde{\phi}}$, then, there exists a positive integer $M$ such that

$$
\widetilde{\phi}\left(x_{\sigma^{m}(u)}-x_{\langle m, n\rangle}\right) \leq M
$$


for all $m$ and $u$. Given $\varepsilon>0$, we get

$$
\begin{aligned}
\frac{1}{g\left(h_{r}\right)} \sum_{m \in I_{r}} \widetilde{\phi}\left(x_{\sigma^{m}(u)}-x_{\langle m, n\rangle}\right) & =\frac{1}{g\left(h_{r}\right)} \sum_{\widetilde{\phi}\left(x_{\left.\sigma^{m}(u)-x_{\langle m, n\rangle}\right) \geq \frac{\varepsilon}{2}}\right.} \widetilde{\phi}\left(x_{\sigma^{m}(u)}-x_{\langle m, n\rangle}\right) \\
& +\frac{1}{g\left(h_{r}\right)} \sum_{\widetilde{t \in I_{r}}} \widetilde{\phi}\left(x_{\sigma^{m}(u)}-x_{\langle m, n\rangle}\right) \\
& \leq \frac{M}{g\left(h_{r}\right)}\left|\left\{m \in I_{r}: \widetilde{\phi}\left(x_{\sigma^{m}(u)}-x_{\langle m, n\rangle}\right) \geq \frac{\varepsilon}{2}\right\}\right|+\frac{\varepsilon}{2} .
\end{aligned}
$$

from which the result follows.

Let $\theta=\left\{k_{r}\right\}$ be given and define $x_{m}$ to be $1,2, \ldots,\left[\sqrt{h_{r}}\right]$ for $m=\sigma^{t}(u), t=$ $k_{r-1}+1, k_{r-1}+2, \ldots, k_{r-1}+\left[\sqrt{h_{r}}\right] ; u \geq 1$, and $x_{m}=0$ otherwise (where [.] denotes the greatest integer function). Note that $x$ is not bounded. Further, for $0<\varepsilon<1$ we get

$$
\frac{1}{g\left(h_{r}\right)}\left|\left\{m \in I_{r}: \widetilde{\phi}\left(x_{\sigma^{m}(u)}-0\right) \geq \varepsilon\right\}\right|=\frac{\left[\sqrt{h_{r}}\right]}{g\left(h_{r}\right)} \rightarrow 0 \text { as } r \rightarrow \infty
$$

i.e. $x_{m} \rightarrow 0\left(A S_{\sigma \theta} C\right)(\widetilde{\phi})^{g}$. But

$$
\frac{1}{g\left(h_{r}\right)} \sum_{m \in I_{r}} \widetilde{\phi}\left(x_{\sigma^{m}(u)}-0\right)=\frac{1}{g\left(h_{r}\right)}\left(\frac{\left[\sqrt{h_{r}}\right]\left(\left[\sqrt{h_{r}}\right]+1\right)}{2}\right) \rightarrow \frac{1}{2} \neq 0 \text { as } r \rightarrow \infty,
$$

hence, $x_{m} \nrightarrow 0\left(A L_{\sigma \theta}\right)(\widetilde{\phi})^{g}$. Thus, inclusion (i) is proper and this example denotes that the boundedness condition can not be omitted from the hypothesis (ii).

(iii) This is an immediate consequence of (i) and (ii).

We now give a lemma which will be used in the proof of Theorem 3.11.

Lemma 3.10. Let $\widetilde{\phi}: \mathbb{R} \rightarrow \mathbb{R}$ be an Orlicz function. Assume for given $\varepsilon_{1}>0$ and every $\varepsilon>0$, there exists $t_{0}$ and $u_{0}$ and there is an integer $n$ such that

$$
\frac{1}{g(t)}\left|\left\{0 \leq m \leq t-1: \widetilde{\phi}\left(x_{\sigma^{m}(u)}-x_{\langle m, n\rangle}\right) \geq \varepsilon\right\}\right|<\varepsilon_{1}
$$

for all $t \geq t_{0}$ and $u \geq u_{0}$, then, $x=\left(x_{m}\right) \in A S_{\sigma} C(\widetilde{\phi})^{g}$.

Proof. Let $\varepsilon_{1}>0$ be given. For each $\varepsilon>0$, select $t_{0}^{\prime}, u_{0}$ such that

$$
\frac{1}{g(t)}\left|\left\{0 \leq m \leq t-1: \widetilde{\phi}\left(x_{\sigma^{m}(u)}-x_{\langle m, n\rangle}\right) \geq \varepsilon\right\}\right|<\frac{\varepsilon_{1}}{2}
$$

for all $t \geq t_{0}^{\prime}$ and $u \geq u_{0}$. It is enough to prove that there exists $t_{0}^{\prime \prime}$ such that $t \geq t_{0}^{\prime \prime}$ and $0 \leq u \leq u_{0}$,

$$
\frac{1}{t}\left|\left\{0 \leq m \leq t-1: \widetilde{\phi}\left(x_{\sigma^{m}(u)}-x_{\langle m, n\rangle}\right) \geq \varepsilon\right\}\right|<\varepsilon_{1} .
$$

Since taking $t_{0}=\max \left\{t_{0}^{\prime}, t_{0}^{\prime \prime}\right\}$, (2) will hold for $t \geq t_{0}$ and for all $u$, which gives the result.

Once $u_{0}$ has been choosen, $0 \leq u \leq u_{0}, u_{0}$ is fixed. So put

$$
S=\left|\left\{0 \leq m \leq u_{0}-1: \widetilde{\phi}\left(x_{\sigma^{m}(u)}-x_{\langle m, n\rangle}\right) \geq \varepsilon\right\}\right| .
$$


Now taking $0 \leq u \leq u_{0}$ and $t \geq u_{0}$, (1) we have

$$
\begin{aligned}
& \frac{1}{g(t)}\left|\left\{0 \leq m \leq t-1: \widetilde{\phi}\left(x_{\sigma^{m}(u)}-x_{\langle m, n\rangle}\right) \geq \varepsilon\right\}\right| \\
& \leq \frac{1}{g(t)}\left|\left\{0 \leq m \leq u_{0}-1: \widetilde{\phi}\left(x_{\sigma^{m}(u)}-x_{\langle m, n\rangle}\right) \geq \varepsilon\right\}\right| \\
& +\frac{1}{g(t)}\left|\left\{u_{0} \leq m \leq t-1: \widetilde{\phi}\left(x_{\sigma^{m}(u)}-x_{\langle m, n\rangle}\right) \geq \varepsilon\right\}\right| \\
& \leq \frac{S}{g(t)}+\frac{1}{g(t)}\left|\left\{u_{0} \leq m \leq t-1: \widetilde{\phi}\left(x_{\sigma^{m}(u)}-x_{\langle m, n\rangle}\right) \geq \varepsilon\right\}\right| \\
& \leq \frac{S}{g(t)}+\frac{\varepsilon_{1}}{2},
\end{aligned}
$$

and taking $t$, sufficiently large, we can write $\frac{S}{g(t)}+\frac{\varepsilon_{1}}{2}<\varepsilon_{1}$, which gives (2), and therefore, the result follows.

Theorem 3.11. Let $\widetilde{\phi}: \mathbb{R} \rightarrow \mathbb{R}$ be an Orlicz function. $A S_{\sigma \theta} C(\widetilde{\phi})^{g}=A S_{\sigma} C(\widetilde{\phi})^{g}$ for every lacunary sequence $\theta$.

Proof. Let $x \in A S_{\sigma \theta} C_{(\widetilde{\phi})^{g}}$. Then, from Definition 3.5, given $\varepsilon_{1}>0$, there exist $r_{0}$ and $x_{\langle m, n\rangle}$ such that

$$
\frac{1}{g\left(h_{r}\right)}\left|\left\{0 \leq m \leq h_{r}-1: \widetilde{\phi}\left(x_{\sigma^{m}(u)}-x_{\langle m, n\rangle}\right) \geq \varepsilon\right\}\right|<\varepsilon_{1}
$$

for $r \geq r_{0}$ and $u=k_{r-1}+1+u, u \geq 0$.

Let $g(t) \geq g\left(h_{r}\right)$, write $g(t)=j g\left(h_{r}\right)+q$, where $0 \leq q<g\left(h_{r}\right), j$ is an integer. Since $g(t) \geq g\left(h_{r}\right), j \geq 1$. Hence we have

$$
\begin{aligned}
& \frac{1}{g(t)}\left|\left\{0 \leq m \leq t-1: \widetilde{\phi}\left(x_{\sigma^{m}(u)}-x_{\langle m, n\rangle}\right) \geq \varepsilon\right\}\right| \\
& \leq \frac{1}{g(t)}\left|\left\{0 \leq m \leq g(t)-1: \widetilde{\phi}\left(x_{\sigma^{m}(u)}-x_{\langle m, n\rangle}\right) \geq \varepsilon\right\}\right| \\
& \leq \frac{1}{g(t)}\left|\left\{0 \leq m \leq(j+1) g\left(h_{r}\right)-1: \widetilde{\phi}\left(x_{\sigma^{m}(u)}-x_{\langle m, n\rangle}\right) \geq \varepsilon\right\}\right| \\
& \leq \frac{1}{g(t)} \sum_{k=0}^{j}\left|\left\{k g\left(h_{r}\right) \leq m \leq(k+1) g\left(h_{r}\right)-1: \widetilde{\phi}\left(x_{\sigma^{m}(u)}-x_{\langle m, n\rangle}\right) \geq \varepsilon\right\}\right| \\
& \leq \frac{g\left(h_{r}\right)}{g(t)}(j+1) \varepsilon_{1} \leq 2 \frac{g\left(h_{r}\right)}{g(t)} j \varepsilon_{1}, \quad(j \geq 1)
\end{aligned}
$$

and since $\frac{j g\left(h_{r}\right)}{g(t)} \leq 1$,

$$
\frac{1}{g(t)}\left|\left\{0 \leq m \leq t-1: \widetilde{\phi}\left(x_{\sigma^{m}(u)}-x_{\langle m, n\rangle}\right) \geq \varepsilon\right\}\right| \leq 2 \varepsilon_{1} .
$$

Then, by the Lemma $1, A S_{\sigma \theta} C_{(\widetilde{\phi})^{g}} \subset A S_{\sigma} C(\widetilde{\phi})^{g}$. It is easy to see that $A S_{\sigma} C(\widetilde{\phi})^{g} \subset$ $A S_{\sigma \theta} C_{(\widetilde{\phi})^{g}}$.

By using the same techniques as in Theorem 3.11, we can prove the following theorem. 
Theorem 3.12. Let $\widetilde{\phi}: \mathbb{R} \rightarrow \mathbb{R}$ be an Orlicz function. $A L_{\sigma \theta}(\widetilde{\phi})^{g} \Leftrightarrow\left[A V_{\sigma}\right](\widetilde{\phi})^{g}$ for every lacunary sequence $\theta$.

If we take $\sigma(u)=u+1$, the above Definitions $3.1-3.7$ reduce the following definitions :

Definition 3.13. A sequence $x=\left(x_{m}\right)$ is said to be almost arithmetic $S$-convergent of weight $g$ if for an integer $n$

$$
\lim _{t \rightarrow \infty} \frac{1}{g(t)} \sum_{m=1}^{t} x_{m+u}=x_{\langle m, n\rangle},
$$

uniformly in $u=1,2, \ldots$.

Definition 3.14. Let $\widetilde{\phi}: \mathbb{R} \rightarrow \mathbb{R}$ be an Orlicz function. A sequence $x=\left(x_{m}\right)$ is said to be strongly almost arithmetic $S(\widetilde{\phi})$-convergent of weight $g$ if for an integer $n$

$$
\lim _{t \rightarrow \infty} \frac{1}{g(t)} \sum_{m=1}^{t} \tilde{\phi}\left(x_{m+u}-x_{\langle m, n\rangle}\right)=0,
$$

uniformly in $u=1,2, \ldots$.

Definition 3.15. Let $\widetilde{\phi}: \mathbb{R} \rightarrow \mathbb{R}$ be an Orlicz function. A sequence $x=\left(x_{m}\right)$ is said to be almost arithmetic weight g-statistically $S(\widetilde{\phi})$-convergent if for every $\varepsilon>0$, there is an integer $n$ such that

$$
\lim _{t \rightarrow \infty} \frac{1}{g(t)}\left|\left\{m \leq t: \widetilde{\phi}\left(x_{m+u}-x_{\langle m, n\rangle}\right) \geq \varepsilon\right\}\right|=0,
$$

uniformly in $u=1,2, \ldots$.

Definition 3.16. Let $\widetilde{\phi}: \mathbb{R} \rightarrow \mathbb{R}$ be an Orlicz function. A sequence $x=\left(x_{m}\right)$ is called to be lacunary almost arithmetic weight g-statistical $S(\widetilde{\phi})$-convergent if for every $\varepsilon>0$, there is an integer $n$ such that

$$
\lim _{r \rightarrow \infty} \frac{1}{g\left(h_{r}\right)}\left|\left\{m \in I_{r}: \widetilde{\phi}\left(x_{m+u}-x_{\langle m, n\rangle}\right) \geq \varepsilon\right\}\right|=0,
$$

uniformly in $u=1,2, \ldots$.

Definition 3.17. Let $\widetilde{\phi}: \mathbb{R} \rightarrow \mathbb{R}$ be an Orlicz function. A sequence $x=\left(x_{m}\right)$ is said to be strongly lacunary almost arithmetic convergent $S(\widetilde{\phi})$-convergent of weight $g$ if for an integer $n$

$$
\lim _{r \rightarrow \infty} \frac{1}{g\left(h_{r}\right)} \sum_{m \in I_{r}} \widetilde{\phi}\left(x_{m+u}-x_{\langle m, n\rangle}\right)=0,
$$

uniformly in $u=1,2, \ldots$

In case $\sigma(u)=u+1$, we have the following statement.

Remark. Similar inclusions to Theorems 3.9, 3.11 and 3.12 hold between lacunary almost arithmetic $\widetilde{\phi}$-convergent of weight $g$ sequences, strongly lacunary almost arithmetic weight g-statistical $\widetilde{\phi}$-convergent sequences, almost arithmetic weight $g$ statistically $\widetilde{\phi}$-convergent sequences and strongly almost aritmetic $\widetilde{\phi}$-convergent of weight g sequences. 
Acknowledgments. The abstract of this work was presented in the "1-st Western Balkan Conference in Mathematics and Applications (WBCMA2021)" which was held between 10-12 June 2021 in Prishtina/Kosovo.

\section{REFERENCES}

[1] A.A. Adem, M. Altmok, Weighted statistical convergence of real valued sequences, Facta Univ. Ser. Math. Inform. 353 (2020) 887-898.

[2] M. Balcerzak, P. Das, M. Filipczak, J. Swaczyna, Generalized kinds of density and the associated ideals, Acta Math. Hungar. 1471 (2015) 97-115.

[3] F. Başar, Summability Theory and its Applications, Bentham Science Publishers, İstanbul, 2012.

[4] F. Başar, H. Dutta, Summable Spaces and Their Duals, Matrix Transformations and Geometric Properties, CRC Press, Taylor \& Francis Group, Monographs and Research Notes in Mathematics, Boca Raton, London, New York, 2020.

[5] C. Belen, S.A. Mohiuddine, Generalized weighted statistical convergence and application, Appl. Math. Comput. 21918 (2013) 9821-9826.

[6] N.L. Braha, H.M. Srivastava, S.A. Mohiuddine, A Korovkin's type approximation theorem for periodic functions via the statistical summability of the generalized de la Vallée Poussin mean, Appl. Math. Comput. 228 (2014) 162-169.

[7] R. Çolak, Lacunary strongly convergence of difference sequences with respect to a modulus function, Filomat 17 (2003) 9-14.

[8] P. Das, E. Savaş, S. Ghosal, On generalized of certain summability methods using ideals, Appl. Math. Lett. 24 (2011) 1509-1514.

[9] H. Dutta, F. Başar, A generalization of Orlicz sequence spaces by Cesàro mean of order one, Acta Math. Univ. Comenianae 2 (2011) 185-200.

[10] I.A. Demirci, M. Gürdal, Lacunary statistical convergence for sets of triple sequences via Orlicz function, Theory Appl. Math. Comput. Sci. 111 (2021) 1-13.

[11] I.A. Demirci, M. Gürdal, On deferred statistical convergence for the sets of triple sequences, J. Math. Anal. 124 (2021) 38-50.

[12] H. Fast, Sur la convergence statistique, Colloq. Math. 2 (1951) 241-244.

[13] A.R. Freedman, J.J. Sember, M. Raphael, Some Cesàro-type summability spaces, Proc. Lond. Math. Soc. 37 (1978) 508-520.

[14] J.A. Fridy, On statistical convergence, Analysis (Munich) 5 (1985) 301-313.

[15] J.A. Fridy, C. Orhan, Lacunary statistical convergence, Pacific J. Math. 1601 (1993) 43-51.

[16] M. Gürdal, Some types of convergence. Doctoral Dissertation. S. Demirel Univ. Isparta, 2004.

[17] M. Gürdal, M.B. Huban, Statistical convergence and $C^{*}$-operator algebras, Theory Appl. Math. Comput. Sci. 7 (2017) 41-50.

[18] M. Gürdal, S. Pehlivan, Statistical convergence in 2-normed spaces, Southeast Asian Bull. Math. 332 (2009) 257-264.

[19] M.B. Huban, Generalized statistically pre-Cauchy triple sequences via Orlicz functions, J. Nonlinear Sci. Appl. 146 (2021) 414-422.

[20] M.B. Huban, M. Gürdal, Wijsman Lacunary invariant statistical convergence for triple sequences via Orlicz function, J. Class. Anal. 221 (2021) 119-128.

[21] M.B. Huban, M. Gürdal, H. Baytürk, On asymptotically lacunary statistical equivalent triple sequences via ideals and Orlicz function, Honam Math. J. 432 (2021) 343-357.

[22] U. Kadak, S.A. Mohiuddine, Generalized statistically almost convergence based on the difference operator which includes the $(p, q)$-gamma function and related approximation theorems, Results Math. (2018), 73:9.

[23] Ö. Kişi, On $\mathcal{I}_{\sigma}$ arithmetic convergence, Malaya J. Mat. 93 (2021) 64-71.

[24] Ö. Kişi, On lacunary $\mathcal{I}$-invariant arithmetic convergence, Malaya J. Mat. 92 (2021) 1-11.

[25] Ö. Kişi, On I-lacunary arithmetic statistical convergence, J. Appl. Math. Inform., in press (2021).

[26] J. Li, Lacunary statistical convergence and inclusion properties between lacunary methods, Internat. J. Math. Math. Sci. 233 (2000) 175-180.

[27] S.A. Mohiuddine, B.A.S. Alamri, Generalization of equi-statistical convergence via weighted lacunary sequence with associated Korovkin and Voronovskaya type approximation theorems, Rev. R. Acad. Cienc. Exactas Fis. Nat. Ser. A Math. RACSAM 1133 (2019) 1955-1973. 
[28] M. Mursaleen, F. Başar, Sequence Spaces: Topics in Modern Summability Theory, CRC Press, Taylor \& Francis Group, Series: Mathematics and Its Applications, Boca Raton, London, New York, 2020.

[29] A. A. Nabiev, E. Savaş, M. Gürdal, Statistically localized sequences in metric spaces, J. Appl. Anal. Comput. 92 (2019) 739-746.

[30] N. Pancaroğlu, F. Nuray, Statistical lacunary invariant summability, Theoretical Math. Appl. 32 (2013), 71-78.

[31] M. M. Rao, Z. D. Ren, Applications of Orlicz spaces, Marcel Dekker Inc., 2002.

[32] R.A. Raimi, Invariant means and invariant matrix methods of summability, Duke Math. J. 30 (1963) 81-94.

[33] W.H. Ruckle, Arithmetical summability, J. Math. Anal. Appl. 396 (2012) 741-748.

[34] T. Salát, On statistically convergent sequences of real numbers, Math. Slovaca 30 (1980) 139-150.

[35] E. Savaş, On I-lacunary statistical convergence of weight $g$ of sequences of sets, Filomat 31 16 (2017) 5315-5322.

[36] E. Savaş, P. Das, A generalized statistical convergence via ideals, Appl. Math. Lett. 24 (2011) 826-830.

[37] E. Savaş, S. Debnath, Lacunary statistically $\phi$-convergence, Note Mat. 392 (2019) 111-119.

[38] E. Savaş, F. Nuray, On $\sigma$-statistically convergence and lacunary $\sigma$-statistically convergence, Math. Slovaca 433 (1993) 309-315.

[39] E. Savaş, U. Yamanc1, M. Gürdal, I-lacunary statistical convergence of weighted $g$ via modulus functions in 2-normed spaces, Commun. Fac. Sci. Univ. Ank. Sér. A1 Math. Stat. 682 (2019) 2324-2332.

[40] P. Schaefer, Infinite matrices and invariant means, Proc. Amer. Math. Soc. 361 (1972) 104-110.

[41] T. Yaying, B. Hazarika, On arithmetical summability and multiplier sequences, Natl. Acad. Sci. Lett. 401 (2017) 43-46.

[42] T. Yaying, B. Hazarika, Lacunary arithmetical statistical convergence, Natl. Acad. Sci. Lett. 436 (2020) 547-551.

Mualla Birgül Huban

Isparta University of Applied Sciences, Isparta, Turkey

E-mail address: muallahuban@isparta.edu.tr

Mehmet GürdAL

Department of Mathematics, Suleyman Demirel University, 32260, Isparta, Turkey

E-mail address: gurdalmehmet@sdu.edu.tr 